\title{
Evolução da resistência de Klebsiella pneumoniae no Hospital Universitário de Londrina no período de 2000 a 2011
}

\author{
Evolution of resistance of Klebsiella pneumoniae in Londrina \\ University Hospital from 2000 to 2011
}

\author{
Diogo Jorge Rossi ${ }^{1}$; Daniele Zendrini Rechenchoski²; Ana Carolina Polano Vivan ${ }^{3}$; \\ Angélica Marim Lopes Dambróziơ; Renata Perugini Biasi Garbin ${ }^{5}$; Gerusa Luciana \\ Gomes Magalhães ${ }^{6}$; Regina Mariuza Borsato Quesada ${ }^{7}$; Floristher Elaine Carrara \\ Marroni ${ }^{8}$; Marsileni Pelisson'; Marcia Regina Eches Perugini ${ }^{10}$; Eliana Carolina
}

Resumo

O presente estudo teve como objetivo avaliar a evolução da resistência a antimicrobianos em isolados clínicos de Klebsiella pneumoniae, no período de 2000 a 2011, no Hospital Universitário da Universidade Estadual de Londrina (HU-UEL). Foi realizada uma análise retrospectiva de 2.318 testes de sensibilidade aos antimicrobianos de K. pneumoniae, a partir de um banco de dados do setor de Microbiologia do Laboratório Clínico do HU. No período de 2000 a 2009, o principal mecanismo de resistência aos antimicrobianos $\beta$-lactâmicos observado foi a produção de $\beta$-lactamases do tipo ESBL ( $\beta$-lactamase de espectro ampliado), que pode ser verificado pelo aumento da resistência de $K$. pneumoniae às cefalosporinas de $3^{\mathrm{a}}$. e $4^{\mathrm{a}}$. geração. No entanto, a partir de 2009 apareceram as primeiras cepas de K. pneumoniae produtoras de carbapenemase, comprometendo a eficácia dos carbapenêmicos. Os índices de resistência ao ertapenem variaram de $16 \%$, em 2005, para 40\%, em 2011. Outra classe de antimicrobianos comprometida foi a das fluoroquinolonas; para ciprofloxacina, os índices de resistência variaram de 13\% a 62\%, em 2001 e 2011, respectivamente. Os aminoglicosídeos tiveram oscilações de resistência durante o período estudado, chegando, em 2011, a valores de $56 \%$ e $30 \%$ para gentamicina e amicacina, respectivamente. Enquanto isso, sulfametoxazol/ trimetoprim e piperacilina/tazobactam alcançaram índices de resistênciade $60 \%$, nesse mesmo período. $\mathrm{O}$ aumento de resistência em $K$. pneumoniae neste hospital evidencia a necessidade de adequação do tratamento de infecções por este agente e de adoção de medidas apropriadas que visem ao controle de infecções, bem como ao uso adequado dessas drogas.

Palavras-chave: Klebsiella pneumoniae. ESBL. KPC. Resistência antimicrobiana.

${ }^{1}$ Graduando em Medicina pela Universidade Estadual de Londrina, Brasil.

${ }^{2}$ Graduação em Farmácia e Mestrado em Microbiologia pela Universidade Estadual de Londrina, Brasil.

${ }^{3}$ Graduação em Farmácia e Mestrado em Microbiologia pela Universidade Estadual de Londrina, Brasil.

${ }^{4}$ Graduação em Farmácia e Mestrado em Microbiologia pela Universidade Estadual de Londrina, Brasil.

5 Graduação em Farmácia pela Universidade Estadual de Maringa e Mestrado em Microbiologia pela Universidade Estadual de Londrina, Brasil .

${ }^{6}$ Especialização em Controle de Infecção Hospitalar pela Universidade Estadual de Londrina, Brasil.

${ }^{7}$ Graduação em Farmácia Bioquímica pela Universidade Estadual de Londrina e Mestrado em Farmácia (Análises Clínicas) pela

${ }^{8}$ Universidade de São Paulo.

${ }^{9}$ Doutorado em Microbiologia pela Universidade Estadual de Londrina, Brasil.

${ }^{10}$ Mestrado em Microbiologia pela Universidade Estadual de Londrina, Brasil.

${ }^{11}$ Doutorado em Doenças Infecciosas e Parasitárias pela Universidade de São Paulo, Brasil.

${ }^{12}$ Doutorado em Microbiologia pela Universidade Estadual de Londrina, Brasil. 


\begin{abstract}
The present study aimed to evaluate the evolution of antibiotic resistance in clinical isolates of Klebsiella pneumoniae in the period of 2000 to 2011, at the University Hospital of Londrina (HU-UEL). A retrospective analysis of 2,318 antimicrobial susceptibility tests of Klebsiella pneumoniae was performed from a database of the Clinical Laboratory of Microbiology of the University Hospital. In the period of 2000 to 2009 , the main mechanism of resistance observed to $\beta$-lactam antimicrobials was due to the production of ESBL $\beta$-lactamase type ( $\beta$-lactamase wide spectrum), which can be verified by the increased resistance of Klebsiella pneumoniae to 3rd generation cephalosporins and cefepime. However, the first strains of Klebsiella pneumoniae carbapenemase-producing appeared in 2009, compromising the efficacy of carbapenems. The rates of resistance to ertapenem ranged from $16 \%$, in 2005 , to $40 \%$ in 2011 . Another class of committed antibiotics was the fluoroquinolones; for ciprofloxacin, resistance rates ranged from $13 \%$ to $62 \%$, in 2001 and 2011, respectively. Aminoglycosides exhibited oscillations of resistance during the period studied, reaching, in 2011, values of $56 \%$ and $30 \%$ for gentamicin and amikacin, respectively. Meanwhile, trimethoprim/ sulfamethoxazole and piperacillin/tazobactam reached about $60 \%$, in the same period. Therefore, knowing the antimicrobial resistance of Klebsiella pneumoniae strains is essential for proper treatment of patients and adoption of appropriate measures that aims infection control, and proper use of these drugs.
\end{abstract}

Keywords: Klebsiella pneumonia. ESBL. KPC. Antimicrobial resistance.

\section{Introdução}

A emergência e a disseminação de resistência entre as espécies da família Enterobacteriaceae trazem complicações para o tratamento de infecções hospitalares e podem contribuir para o aparecimento de espécies resistentes a todos antimicrobianos atualmente disponíveis. Em particular, K. pneumoniae é um patógeno extremamente importante em infecções hospitalares, uma vez que pode apresentar resistência múltipla a antimicrobianos de diferentes classes (PATERSON, 2006).

A facilidade de colonizar mucosa privilegia $K$. pneumoniae como patógeno oportunista. Infecções por este agente comprometem, principalmente, o trato urinário e o trato respiratório, que podem levar à bacteremia grave e à pneumonia aspirativa, com altas taxas de morbidade e mortalidade. Entre a variedade de infecções extrapulmonares associadas a K. pneumoniae, a infecção urinária, tanto em crianças quanto em adultos, é a mais prevalente. Esta espécie também pode causar meningite, endocardite, infecções dos tecidos moles e feridas cutâneas (SCARPATE; COSSATIS, 2009; PODSCHUN; ULLMANN, 1998).

O principal mecanismo de resistência de bactérias Gram-negativas é a produção de $\beta$-lactamases, enzimas que inibem a ação de drogas $\beta$-lactâmicas, impossibilitando assim a sua atividade antimicrobiana (LIVERMORE, 1998). Em Klebsiella spp., as $\beta$-lactamases de amplo espectro (ESBL) desempenham importante papel na resistência a antimicrobianos. ESBL são enzimas capazes de hidrolisar todos os antimicrobianos $\beta$-lactâmicos, com exceção das cefamicinas, inibidores de $\beta$-lactamases e carbapenêmicos. As espécies produtoras de ESBL podem sobreviver por longos períodos em hospitais, com frequência ocasionando surtos (BRADFORD, 2001; THOMSON; PREVAN; SANDERS, 1996).

Os carbapenêmicos são agentes antimicrobianos extremamente utilizados na atualidade e, muitas vezes, o último recurso para o tratamento de infecções graves causadas por enterobactérias produtoras de ESBL (NICOLAU, 2008; PATERSON et al., 2004; PITOUT; LAUPLAND, 2008; TUMBARELLO et al., 2007). Recentemente, entretanto, a resistência aos carbapenêmicos tem emergido e se disseminado em uma escala global, o que representa um sério desafio para a saúde pública, pois compromete as opções terapêuticas e o controle de infecções hospitalares (CARMELI et al., 2010; MIRIAGOU et al., 2010).

A carbapenemase é a enzima que confere resistência aos carbapenêmicos, configurando-se 
como importante mecanismo de resistência em ambiente hospitalar. As infecções causadas por micro-organismos produtores de carbapenemases estão associadas com altas taxas de morbidade e mortalidade (DIENSTMANN et al., 2010).

No Brasil, a primeira cepa de $K$. pneumoniae produtora da carbapenemase do tipo K. pneumoniae carbapenemase (KPC) foi descrita em 2006, no Recife. Um estudo retrospectivo demonstrou que este mecanismo de resistência esteve presente nos hospitais brasileiros desde, pelo menos, 2005, embora não tivesse sido detectado nessa época (PAVEZ; MAMIZUKA; LINCOPAN, 2009). Hoje em dia, bactérias produtoras de KPC têm sido detectadas em várias regiões brasileiras (ZAVASCKI et al., 2010) .

No HU-UEL foi detectado um surto por K.pneumoniae produtora de KPC em 2009. Sabendose que o surgimento deste mecanismo de resistência traz inúmeras complicações no tratamento de infecções graves e que estes micro-organismos frequentemente apresentam multirresistência, o presente trabalho teve como objetivo avaliar a evolução da resistência produzida por $K$. pneumoniae a diversas classes de antimicrobianos, no período de 2000 a 2011, no HU-UEL.

\section{Materiais e Métodos}

Foi realizado um estudo descritivo retrospectivo através da análise de resultados de testes de sensibilidade a antimicrobianos (TSA) em 2.318 isolados clínicosde K.pneumoniae obtidos depacientes atendidos e internados no HU-UEL, de janeiro de
2000 a dezembro de 2011. Os dados avaliados foram obtidos de um banco de dados cadastrados no sistema de informação AGTA Healthcare, módulo Labhos(R), do setor, do setor de Microbiologia do Laboratório de Análises Clínicas do HU-UEL.

A identificação e o teste de sensibilidade a antimicrobianos foram realizados por metodologia automatizada, utilizando-se os sistemas MicroScan ${ }^{\circledR}$ (Siemens) ou BD Phoenix ${ }^{\circledR}$ (Becton Dickinson). Quando necessário, foram realizados testes fenotípicos para confirmação de ESBL, como descrito por Jarlier e colaboradores (1988). Foram avaliados os seguintes antimicrobianos: cefalotina, cefotaxima, ceftazidima, cefepime, aztreonam, imipenem, meropenem, ertapenem, piperacilina/ tazobactam, amicacina, gentamicina, ciprofloxacina, levofloxacina, nitrofurantoína e sulfametoxazol/ trimetoprim.

\section{Resultados e Discussão}

A resistência bacteriana representa um grande desafio no tratamento de diversas infecções, principalmente as causadas por bactérias produtoras de ESBL e, mais recentemente, as enterobactérias produtoras de carbapenemases, em especial as cepas de K. pneumoniae.

Analisando-se os dados obtidos neste estudo, pode-se verificar que ocorreu um aumento de resistência à maioria dos antimicrobianos avaliados, com destaque para os $\beta$-lactâmicos, como mostra a tabela 1 . 
Tabela 1 - Frequência de resistência (\%) de 2.318 isolados clínicos de K. pneumoniae aos $\beta$-lactâmicos, no período de janeiro de 2000 a dezembro de 2011, em pacientes internados no HU-UEL

\begin{tabular}{lccccccccccccc}
\hline Antibióticos/Ano & $\mathbf{2 0 0 0}$ & $\mathbf{2 0 0 1}$ & $\mathbf{2 0 0 2}$ & $\mathbf{2 0 0 3}$ & $\mathbf{2 0 0 4}$ & $\mathbf{2 0 0 5}$ & $\mathbf{2 0 0 6}$ & $\mathbf{2 0 0 7}$ & $\mathbf{2 0 0 8}$ & $\mathbf{2 0 0 9}$ & $\mathbf{2 0 1 0}$ & $\mathbf{2 0 1 1}$ & Média \\
\hline CFL & 18 & 28 & 44 & 56 & 65 & 54 & 63 & 36 & 57 & 61 & 61 & 68 & 51 \\
CTX & 7 & 11 & 14 & 18 & 38 & 38 & 45 & 36 & 42 & 47 & 58 & 60 & 35 \\
CAZ & 6 & 5 & 13 & 13 & 25 & 27 & 33 & 25 & 31 & 42 & 58 & 59 & 28 \\
CFM & 16 & 12 & 35 & 46 & 49 & 42 & 48 & 38 & 43 & 47 & 58 & 60 & 41 \\
ATM & 34 & 11 & 17 & 18 & 37 & 35 & 45 & 33 & 39 & 47 & 58 & 60 & 36 \\
IMI & 0 & 0 & 0 & 0 & 0 & 0 & 0 & 0 & 0 & 6 & 27 & 28 & 5 \\
MER & 0 & 0 & 0 & 0 & 0 & 0 & 0 & 0 & 0 & 11 & 34 & 32 & 6 \\
ERT & 0 & 0 & 0 & 0 & 0 & 16 & 13 & 13 & 18 & 26 & 43 & 40 & 14 \\
PIP/TAZ & 48 & 34 & 37 & 46 & 44 & 41 & 42 & 37 & 45 & 43 & 58 & 60 & 45 \\
\hline
\end{tabular}

CFL: cefalotina; CTX: cefotaxima; CAZ: ceftazidima; CFM: cefepime; ATM: aztreonam; IMI: imipenem; MER: meropenem;, ERT: ertapenem; PIP/TAZ: piperacilina/tazobactam, ERT Fonte: autores

A deteç̧ão de ESBL foi realizada pelos sistemas de automação MicroScan ${ }^{\circledR}$ (Siemens) ou BD Phoenix ${ }^{\circledR}$ (Becton Dickinson). Resultados semelhantes foram relatados em diversos países, dados publicados pelo Study for Monitoring Antimicrobial Resistance Trends, de 2004, demonstraram que a prevalência de ESBL entre as espécies de Klebsiella variaram de 5,3\% nos EUA a 8,8\% na Europa, 27,6\% na América Latina, 27,4\% no Oriente Médio/África e 22,9\% na Ásia/Pacífico (ROSSI et al., 2006). No Brasil, dados de 2011 do Hospital das Clínicas da Universidade de São Paulo, apontam índices de 40 a 50\% de ESBL em isolados de K. pneumoniae (ROSSI, et al. (2011).

Resistência a carbapenêmicos foi detectada em 2005, para ertapenem, com índice de 16\%. Porém, essa resistência ocorria devido a mecanismos não associados à produção de $\beta$-lactamases do tipo carbapenemases, mas já estava despertando a atenção e vigilância da equipe clínica e laboratorial do HUUEL. Em 2009, aproximadamente 14,3\% dos isolados de $K$. pneumoniae apresentavam resistência aos antimicrobianos carbapenêmicos, alcançando índices de 34,6\% em 2010 e 33,3\% em 2011, tornando-se uma preocupação constante no hospital, em virtude de sua ocorrência já ser considerada endêmica na instituição.
Os primeiros isolados de $K$. pneumoniae produtores de KPC, no HU-UEL, ocorreram em um surto em fevereiro de 2009, quando os isolados deste microorganismo começaram a apresentar resistência de $6 \%$ para imipenem e $11 \%$ para meropenem (VESPERO et al., 2009). Em 2011, a resistência a carbapenêmicos alcançou índices de 40\% para ertapenem, 28\% para imipenem e $32 \%$ para meropenem, preocupando a equipe de Controle de Infecção do HU-UEL.

$\mathrm{O}$ aumento da resistência a carbapenêmicos em $K$. pneumoniae também tem sido verificado por outros autores. O estudo conduzido pelo International Nosocomial Infection Control Consortium (INICC), de janeiro de 2004 a dezembro de 2009, envolvendo amostras obtidas de 422 Unidades de Terapia Intensiva (UTI) de 36 países mostrou que $7,9 \%$ dos 508 isolados de $K$. pneumoniae eram resistentes aos carbapenêmicos (ROSENTHAL et al., 2012). Um relatório do programa de vigilância antimicrobiana SENTRY, que analisou 5516 isolados de bactérias Klebsiella spp., em 83 centros médicos de vários continentes, apontou taxas de resistência aos carbapenêmicos e produção de carbapenemase de $5,3 \%$ e $3,8 \%$, respectivamente (CASTANHEIRA et al., 2011). Outro estudo realizado com 15 hospitais do Brooklyn, nos anos de 1999, 2001 e 2006, mostrou 
aumento de resistência a carbapenêmicos. Em 1999, dos 824 isolados, $4 \%, 0,4 \%$ e $0,1 \%$ foram resistentes ao ertapenem, imipenem e meropenem, respectivamente. Enquanto isso, em 2006, dos 997 isolados, 39\%, 24\% e $24 \%$ foram resistentes ao ertapenem, imipenem e meropenem, respectivamente (LANDMAN et al., 2007).

Da mesma forma, observou-se o aumento de resistência para fluoroquinolonas, aminoglicosídeos e sulfonamidas, como pode ser visto na tabela 2. As taxas de resistência à ciprofloxacina aumentaram de $13 \%$, em 2001, para $62 \%$, em 2011 , enquanto que os aminoglicosídeos apresentaram um aumento maior de resistência de 2000 a 2004 , de $22 \%$ a $36 \%$ para amicacina e de $33 \%$ a $58 \%$ para gentamicina, e se mantiveram relativamente estáveis até 2011 (30\% para amicacina e $56 \%$ para gentamicina). A resistência ao sulfametoxazol/trimetoprim também aumentou de cerca de $30 \%$ para $60 \%$ no período analisado.

Tabela 2 - Frequência de resistência (\%) de 2.318 isolados clínicos de K. pneumoniae aos aminoglicosídeos, fluoroquinolonas, nitrofurantoína e sulfametoxazol/trimetoprim, no período de janeiro de 2000 a dezembro de 2011, em pacientes internados no HU-UEL

\begin{tabular}{lccccccccccccc}
\hline Antibióticos/Ano & $\mathbf{2 0 0 0}$ & $\mathbf{2 0 0 1}$ & $\mathbf{2 0 0 2}$ & $\mathbf{2 0 0 3}$ & $\mathbf{2 0 0 4}$ & $\mathbf{2 0 0 5}$ & $\mathbf{2 0 0 6}$ & $\mathbf{2 0 0 7}$ & $\mathbf{2 0 0 8}$ & $\mathbf{2 0 0 9}$ & $\mathbf{2 0 1 0}$ & $\mathbf{2 0 1 1}$ & Média \\
\hline AMI & 22 & 13 & 15 & 23 & 36 & 31 & 32 & 21 & 27 & 31 & 38 & 30 & 27 \\
GEN & 33 & 32 & 33 & 47 & 58 & 46 & 51 & 44 & 48 & 53 & 51 & 56 & 46 \\
CIP & 29 & 13 & 32 & 48 & 55 & 49 & 52 & 48 & 49 & 54 & 63 & 62 & 46 \\
LEV & 70 & 15 & 35 & 52 & 53 & 49 & 50 & 44 & 48 & 52 & 62 & 69 & 50 \\
NIT & 48 & 54 & 64 & 80 & 84 & 76 & 79 & 75 & 67 & 67 & 69 & 68 & 69 \\
STX & 40 & 29 & 40 & 55 & 59 & 51 & 58 & 46 & 48 & 56 & 62 & 59 & 50
\end{tabular}

AMI: amicacina; GEN: gentamicina; CIP: ciprofloxacina; LEV: levofloxacina; NIT: nitrofurantoína; STX: sulfametoxazol/trimetoprim

Fonte: autores

Um estudo em UTI de 14 hospitais holandeses, durante o período de 1998 a 2009, mostrou aumento da resistência de isolados de $K$. pneumoniae às fluoroquinolonas e sulfonamidas. A taxa de resistência a ciprofloxacina aumentou de 5,8\% para $18,5 \%$, enquanto que, para sulfametoxazol/ trimetoprim, a variação foi de $11,9 \%$ para $23,1 \%$ (VAN DER DONK et al., 2011).

A pesquisa de Landman e colaboradores (2007) também mostra índices maiores de resistência as fluoroquinolonas e aos aminoglicosídeos. Neste estudo, as taxas de resistência para a ciprofloxacina variaram de $26 \%$ a $59 \%$ de 1999 a 2006, respectivamente. Para a amicacina, a resistência aumentou de 14\% em 1999 para 46\% em 2006.
O uso abusivo e indiscriminado de antibióticos na comunidade e nos hospitais, seja por má indicação médica ou por automedicação, tem facilitado o surgimento dessas bactérias multirresistentes. Esses micro-organismos adquirem altos níveis de resistência aos antibióticos por meio de mutações nos genes cromossômicos ou pelo intercâmbio de material genético através de elementos genéticos móveis. De modo geral, pode-se definir a evolução da resistência bacteriana como resultado de: fenômeno fisiológico, pressão seletiva, fatores intrínsecos ou naturais e extrínsecos ou adquiridos.

Pode-se concluir que a resistência de $K$. pneumoniae à maioria dos antimicrobianos aumentou no período analisado, no HU de 
Londrina, destacando-se o aumento de resistência aos $\beta$-lactâmicos. O surgimento de resistência a carbapenêmicos é um fato bastante preocupante, pois representa uma limitação terapêutica, uma vez que os carbapenêmicos constituem, na atualidade, opção para o tratamento de cepas produtoras de ESBL.

A aquisição deste e de outros mecanismos de resistência em $K$. pneumoniae, em especial a carbapenêmicos, tem se tornado uma preocupação em nossa instituição, como tem ocorrido em outras partes do mundo. $\mathrm{O}$ conhecimento da mudança de resistência, verificada nos últimos tempos, reforça a necessidade da manutenção de barreiras para prevenção e controle da disseminação desta bactéria no âmbito hospitalar.

\section{Referências}

BRADFORD, P. A. Extended-spectrum $\beta$-lactamase in the 21st century: characterization, epidemiology and detection of this important resistance threat. Clinical Microbiology Reviews, Washington, v. 14, n. 4, p. 933951, 2001.

CARMELI, Y.; AKOVA, M.; CORNAGLIA, G.; DAIKOS, G. L.; GARAU, J.; HARBARTH, S.; ROSSOLINI, G. M.; SOULI, M.; GIAMARELLOU, $\mathrm{H}$. Controlling the spread of carbapenemase-producing Gram-negatives: therapeutic approach and infection control. Clinical Microbiology and Infection, Oxford, v. 16, n. 2, p. 102-11, 2010.

CASTANHEIRA, M.; MENDES, R. E.; WOOSLEY, L. N.; JONES, R. N. Trends in carbapenemase-producing Escherichia coli and Klebsiella spp. from Europe and the Americas: report from the SENTRY antimicrobial surveillance programme (2007-09). Journal of Antimicrobial Chemotherapy, London, v. 66, n. 6, p. 1409-1411, 2011.

DIENSTMANN, R.; PICOLI, S. U.; MEYER, G.; SCHENKEL, T.; STEYER, J. Avaliação fenotípica da enzima Klebsiella pneumoniae carbapenemase (KPC) em Enterobacteriaceae de ambiente hospitalar. Jornal Brasileiro de Patologia e Medicina Laboratorial, Rio de Janeiro, v. 46, n. 1, p. 23-27, 2010.
JARLIER,V.; NICOLAS, M. H.; FOURNIER, G.; PHILIPPON, A. Extended broad-spectrum betalactamases conferring transferable resistance to newer beta-lactam agents in Enterobacteriaceae hospital prevalence and susceptibility patterns. Reviews of Infectious Diseases, Chicago, v. 10, n. 4, p. 867-878, 1988.

LANDMAN, D.; BRATU, S.; KOCHAR, S.; PANWAR, M.; TREHAN, M.; DOYMAZ, M.; QUALE, J. Evolution of antimicrobial resistance among Pseudomonas aeruginosa, Acinetobacter baumannii and Klebsiella pneumoniae in Brooklyn, NY. Journal of Antimicrobial Chemotherapy, London, v. 60, n. 1, p. 7882, 2007.

LIVERMORE, D. $\quad$ M. $\quad \beta$-lactamase-mediated resistance and opportunities for its control. Journal of Antimicrobial Chemotherapy, London, v. 41, n. 4, p. 25-41, 1998.

MIRIAGOU, V.; CORNAGLIA, G.; EDELSTEIN, M.; GALANI, I.; GISKES, C. G.; GNIADKOWSKIS, M.; MALAMOU-LADA, E.; MARTINEZMARTINEZ, L.; NAVARRO, F.; NORDMANN, P.; PEIXE, L.; POURNARAS, S.; ROSSOLINI, G. M.; TSAKRIS, A.; VATOPOULOS, A.; CANTÓN, R. Acquired carbapenemases in Gram-negative bacterial pathogens: detection and surveillance issues. Clinical Microbiology and Infection, Oxford, v. 16, n. 2, p. 112122, 2010.

NICOLAU, D. P. Pharmacokinetic and pharmacodynamic properties of meropenem. Clinical Infectious Diseases, Chicago, v. 47, n. 1, p. 32-40, 2008.

PATERSON, D. L. Resistance in Gram-negative bacteria: enterobacteriaceae. The American Journal of Medicine, New York, v. 119, n. 6, p. 20-28, 2006.

PATERSON, D. L.; KO, W. C.; GOTTBERG, A. V.; MOHAPATRA, S.; CASELLAS, J. M.; GOOSSENS, H.; MULAZIMOGLU, L.; TRENHOLME, G.; KLUGMAN, K. P.; BONOMO, R. A.; RICE, L. B.; WAGENER, M. M.; MCCORMACK, J. G.; YU, V. L. International prospective study of Klebsiella pneumoniae bacteremia: implications of extendedspectrum-lactamase production in nosocomial infections. Annals of Internal Medicine, Philadelphia, v. 140, n. 1, p. 26-32, 2004. 
PAVEZ, M.; MAMIZUKA, E. M.; LINCOPAN, N. Early dissemination of KPC-2-producing Klebsiella pneumoniae strains in Brazil. Antimicrobial Agents and Chemotherapy, Bethesda, v. 53, n. 7, p. 2702, 2009.

PITOUT, J. D.; LAUPLAND, K. B. Extended-spectrum beta-lactamase-producing Enterobacteriaceae: an emerging public-health concern. The Lancet Infectious Diseases, New York, v. 8, n. 3, p. 159-66, 2008.

PODSCHUN, R.; ULLMANN, U. Klebsiella spp. as nosocomial pathogens: epidemiology, taxonomy, typing methods, and pathogenicity factors. Clinical Microbiology Reviews, Washington, v. 11, n. 4, p. 589-603, 1998.

ROSENTHAL, V. D.; BIJIE, H.; MAKI, D. G.; MEHTA, Y.; APISARNTHANARAK, A.; MEDEIROS, E. A.; LEBLEBICIOGLU, H.; FISHER, D.; ÁLVAREZ-MORENO, C.; KHADER, I. A.; MARTÍNEZ, M. R. G.; CUELLAR, L. E.; NAVOA-NG, J. A.; ABOUGAL, R.; GARCELL, H. G.; MITREV, Z.; GARCÍA, M. C. P.; HAMDI, A.; DUEÑAS, L.; CANCEL, E.; GURSKIS, V.; RASSLAN, O.; AHMED, A.; KANJ, S. S.; CHAVARRÍA, U.; MAPP, T.; RAKA, L.; MENG, C. Y.; THU, L. T. A.; GHAZAL, S.; GIKAS, A.; NARVÁEZ, L. P.; MEJÍA, N.; HADJIEVA, N.; ELANBYA, M. O. G.; SIRITT, M. E. G.; JAYATILLEKE, K. International Nosocomial Infection Control Consortium (INICC) report, data summary of 36 countries, for 2004-2009. American Journal of Infection Control, Saint Louis, v. 40, n. 5, p. 396-407, 2012.

ROSSI, F. The challenges of antimicrobial resistance in Brazil. Clinical Infectious Disease, Chicago, v. 52, n. 9, p. 1138-1143, 2011.

ROSSI, F.; BAQUERO, F.; HSUEH, P.; PATERSON, D. L.; BOCHICCHIO, G. V.; SNYDER, T. A.; SATISHCHANDRAN, V.; MCCARROLL, K.; DINUBILE, M. J.; CHOW, J. W. In vitro susceptibilities of aerobic and facultatively anaerobic Gram-negative bacilli isolated from patients with intra-abdominal infections worldwide: 2004 results from SMART (Study for Monitoring Antimicrobial Resistance Trends). Journal of Antimicrobial Chemotherapy, London, v. 58, n. 1, p. 205-210, 2006.
SCARPATE, E. C. B.; COSSATIS, J. J. A presença da Klebsiella pneumoniae produtora de $\beta$-lactamase de espectro estendido no ambiente hospitalar. Saúde e Ambiente, Rio de Janeiro, v. 4, n. 1, p. 1-11, 2009.

THOMSON, K. S.; PREVAN, A. M.; SANDERS, C. C. Novel plasmid-mediated B-lactamases in Enterobacteriaceae: emerging problems for new B-lactam antibiotics. Current Clinical Topics in Infectious Diseases, New York, v. 16, p. 151-163, 1996.

TUMBARELLO, M.; SANGUINETTI, M.; MONTUORI, E.; TRECARICHI, E. M.; POSTERARO, B.; FIORI, B.; CITTON, R.; D'INZEO, T.; FADDA, G.; CAUDA, R.; SPANU, T. Predictors of mortality in patients with bloodstream infections caused by extended-spectrum- $\beta$ lactamase-producing Enterobacteriaceae: importance of inadequate initial antimicrobial treatment. Antimicrobial Agents and Chemotherapy, Bethesda, v. 51, n. 6, p. 1987-1994, 2007.

VAN DER DONK, C. F.; BEISSER, P. S.; HOOGKAMP-KORSTANJE, J. A.; BRUGGEMAN, C. A.; STOBBERINGH, E. E. A 12 year (1998-2009) antibiotic resistance surveillance of Klebsiella pneumoniae collected from intensive care and urology patients in 14 Dutch hospitals. Journal of Antimicrobial Chemotherapy, London, v. 66, n. 4, p. 855-858, 2011.

VESPERO, E. C.; PELISSON, M.; ROCKSTROH, A. C.; PICÃO, R.; MAGAlHÃES, G. L. G.; PERUGINI, M. R. E.; QUESADA, R. M. B.; GALES, A. C. Surto de Klebsiella pneumoniae produtora de KPC em um Hospital Universitário. In: CONGRESSO BRASILEIRO DE MICROBIOLOGIA, 25., 2009, Porto de Galinhas. Anais... Porto de Galinhas, 2009.

ZAVASCKI, A. P.; ZOCCOLI, C. M.; MACHADO, A. B.; OLIVEIRA, K. R.; S UPERTI, S. V.; PILGER, D. A.; CANTARELLI, V. V.; BARTH, A. L. KPC2-producing Klebsiella pneumoniae in Brazil: a widespread threat in waiting? International Journal of Infectious Diseases, Hamilton, v. 14, n. 6, p. 539540, 2010.

Recebido em: 05 ago. 2014. Aceito em: 19 nov. 2014. 
